\title{
Iterative Learning Control for Multiple Point-to-Point Tracking Application
}

\author{
Chris T. Freeman, Zhonglun Cai, Eric Rogers, and Paul L. Lewin
}

\begin{abstract}
This paper considers a general class of linear iterative learning control (ILC) algorithm applied to tracking tasks which require the plant output to reach given points at predetermined time instants, without the specification of intervening reference points. A framework is developed in the frequency-domain in which the reference is updated between trials. It is shown that superior convergence and robustness properties are obtained compared with those associated with using the original class of ILC algorithm to track a prescribed arbitrary reference trajectory satisfying the point-to-point output constraints. Experimental results using a non-minimum phase test facility are presented to illustrate the theoretical findings.
\end{abstract}

Index Terms-Frequency domain analysis, iterative methods, learning control systems, motion control, optimization methods, test facilities.

\section{INTRODUCTION}

I TERATIVE LEARNING CONTROL (ILC) is a technique that is applicable to processes which repeatedly perform a tracking task defined over a finite interval. ILC uses information from previous trials of the task in the construction of the next control input with the aim of successively improving tracking accuracy. In the great majority of cases, the reference trajectory used does not change between trials, and little work has been conducted to derive ILC algorithms in which the repeated operation may consist of a more general objective, which need not comprise the tracking of a static predefined [1]. Those which do exist generally deal with specialized cases and are linked with specific applications (such as gas metal arc welding [2], underwater robotics [3], or liquid slosh in a packaging machine [4]).

However, in many applications, including production line automation, crane positioning, and robotic "pick and place" tasks, the objective is to repeatedly follow a motion profile in which the error is only critical at certain points. A commonly applied technique for such tasks is point-to-point motion control, in which the objective is to ensure that, at a finite set of prescribed time instants, the system output equals a corresponding set of desired values. Point-to-point control strategies typically involve the generation of a suitable motion profile in advance, and then the design of a controller to track it. The most common approach to generating such a profile is Input Shaping which has been applied in a wide variety of ways

Manuscript received March 23, 2010; accepted May 17, 2010. Manuscript received in final form May 25, 2010. Date of publication June 28, 2010; date of current version April 15, 2011. Recommended by Associate Editor S. S. Saab.

The authors are with the School of Electronics and Computer Science, University of Southampton, Southampton, SO17 1BJ, U.K. (e-mail: cf@ecs.soton.ac.uk).

Digital Object Identifier 10.1109/TCST.2010.2051670
[5]-[7], although other approaches have also been used (see [8], [9], and references therein).

The application of ILC in the area of point-to-point motion control offers the potential to benefit from the ability to learn from experience gained over previous trials of the task. The standard ILC framework is clearly able to tackle such problems simply by using any reference which connects the desired points. However in this paper it is shown that additional performance can be gained by removing the unnecessary constraint that the plant follow a predefined output between points. In the proposed approach the reference sections connecting the points are allowed to vary between trials, and improved convergence and robustness properties compared with using a static reference are demonstrated. This in turn leads to improved performance in tracking the critical points.

There have been a small number of cases in which ILC has been applied to point-to-point control. In [10], Hankel ILC is applied to suppress residual vibrations, where controller matrices are determined through command shaping. In [11], an iterative learning scheme is again used for vibration suppression, and the control parameters are updated via an input shaping technique. In [12], a standard ILC controller is first applied but the control gains are chosen to minimise an error norm motivated by the point-to-point positioning operation rather than the tracking error along the task. These applications of ILC to point-to-point tracking, however, first do not involve updating the reference which is static throughout, and second involve the derivation of explicit algorithms rather than a technique which can be applied to an existing ILC scheme. This latter quality is of clear advantage since it removes the need to design, implement, and test a new controller.

The approach developed in this paper is motivated by the authors' recent application of ILC to stroke rehabilitation. Due to the difficulty in obtaining an accurate model and emphasis on patient comfort and minimizing the time spent on tuning, a modular, transparent, approach to controller implementation, and evaluation is demanded. During treatment stroke patients performed repeated trials of an elliptical tracking task whilst electrical stimulation was applied to their triceps [13]. Despite the difficulty associated with the control task, ILC was able to provide accurate tracking, which in turn led to clinically significant improvements in arm function when applied to patients over the course of 18 treatment sessions [14]. By providing a far higher level of tracking performance than previous controllers applied clinically, the ILC scheme established the approach's ability to provide effective treatment.

However, to maximize the potential benefit to stroke patients, the range of movement tasks must be more functional and resemble those needed to perform everyday tasks. Such 
tasks comprise a series of point-to-point movements, examples including picking up objects or pressing buttons, and it is the ability to pass through these points which maximizes the treatment's potential for rehabilitation. In this context high value is placed on: 1) a control structure which can be used with both standard tracking trajectories and point-to-point tasks without alteration of the underlying ILC scheme; 2) use of existing ILC laws which have already been developed and tested with patients; and 3) adding robustness to these laws.

The framework which is developed in this paper addresses these issues by providing the ability to use an existing general class of ILC laws with multiple-point-to-point movements by simply adjusting the reference between trials, without any modification to the original ILC controller or its gains. It thereby can provide a greater degree of flexibility and adaptability to a wide class of ILC algorithms in a simple but effective manner. It will also be shown that the approach increases the robustness to plant uncertainty whilst still ensuring convergence of the error.

This paper is structured as follows. The use of a trial-dependent reference with a general class of ILC algorithms is introduced in Section II, and the reference update technique is then developed in Section III. Convergence and robustness properties of the resulting system are derived in Sections IV and V, respectively. In Section VI the technique is extended for use with multiple point-to-point movements, and experimental results presented in Section VII confirm the efficacy of the approach in practice. The conclusion and future work are presented in Section VIII.

\section{OBJECTIVE-DRIVEN ILC DEVELOPMENT}

The key departure from the standard ILC framework is that the reference is permitted to change between each trial. It will be shown that this can be achieved using a simple method which has the ability to speed up learning when the intention is not to track a fixed reference, but instead require a specified multiple point-to-point movement task be performed. A single-input-single-output (SISO) linear time invariant (LTI) system is considered, and given, on trial $k$, by

$$
\begin{aligned}
x_{k}(t+1) & =A x_{k}(t)+B u_{k}(t) \\
y_{k}(t) & =C x_{k}(t) \quad t \in[0, T]
\end{aligned}
$$

where $A, B$, and $C$ are matrices of suitable dimension, and a sample time of unity is assumed for notational simplicity. Consider a single point-to-point movement, in which, for all $k$, the output, $y_{k}$, must equal 0 at time 0 , and $r_{N}$ at time $T$. An initial reference could be selected in the form of any trajectory which achieves the point-to-point task (e.g., a straight line trajectory connecting 0 , at time 0 to $r_{N}$ at time $T$ ). To achieve the tracking task an ILC algorithm of the general form

$$
u_{k+1}=u_{k}+K e_{k}
$$

will be considered, where $K$ is a suitable linear operator which may be non-causal (this encompasses a large number of algorithms appearing in the literature, see [1] and [15]). However, at the end of the $k$ th trial, $y_{k}$ is taken and instead of calculating $e_{k}=r-y_{k}$, with a fixed reference, $r$, for use in the update (2), the reference is allowed to change, and is replaced with $r_{k+1}$, leading to

$$
e_{k}^{*}=r_{k+1}-y_{k} .
$$

So the ILC law (2) becomes

$$
u_{k+1}=u_{k}+K e_{k}^{*}
$$

This update replaces (2) and will be considered in the analysis which follows. The time domain relationships which arise using (4) are

$$
\begin{aligned}
y_{k+1} & =y_{k}+G K e_{k}^{*} \\
r_{k+1}-y_{k+1} & =r_{k+1}-y_{k}-G K e_{k}^{*} \\
e_{k+1} & =(I-G K)\left(r_{k+1}-y_{k}\right)
\end{aligned}
$$

where $e_{k}=r_{k}-y_{k}$ is the error prior to updating the reference, $I$ is the identity operator, and $G$ is the lifted plant matrix. Therefore the criterion for monotonic convergence of the error (see, for example, [16]) becomes

$$
\left\|e_{k+1}\right\|^{2}=\left\|(I-G K)\left(r_{k+1}-y_{k}\right)\right\|^{2}<\left\|e_{k}\right\|^{2}
$$

and since

$$
\left\|(I-G K)\left(r_{k+1}-y_{k}\right)\right\|^{2} \leq\left\|r_{k+1}-y_{k}\right\|^{2}\|I-G K\|^{2}
$$

a sufficient condition for monotonic convergence is

$$
\|I-G K\|^{2}<1
$$

together with the requirement

$$
\frac{\left\|r_{k+1}-y_{k}\right\|^{2}}{\left\|e_{k}\right\|^{2}}=\frac{\left\|r_{k+1}-y_{k}\right\|^{2}}{\left\|r_{k}-y_{k}\right\|^{2}} \leq 1
$$

which equates to

$$
\left\|r_{k+1}-y_{k}\right\|^{2} \leq\left\|r_{k}-y_{k}\right\|^{2} .
$$

The condition (10) is the monotonic convergence criterion associated with the ILC algorithm (2) using a static reference. If this is satisfied then $r_{k+1}$ must be chosen such that (12) is satisfied. This can also be expressed as

$$
\left\|r_{k+1}-y_{k}\right\|^{2}=\left\|\Delta r_{k}+e_{k}\right\|^{2} \leq\left\|e_{k}\right\|^{2}
$$

where $\Delta r_{k}=r_{k+1}-r_{k}$. The initial reference already satisfies the end-point constraints so it is possible to choose $\Delta r_{k}$ from a suitable set of functions with end-points equal to zero for $k \geq 2$. The end point constraints will then be satisfied over all trials. Here the set of harmonic sinewaves will be considered, since this leads to significant simplification in how $\Delta r_{k}$ is chosen, although it also limits it to be an even function. This choice is equivalent to taking the discrete Fourier transform (DFT) of $\Delta r_{k}$, with components, $\Delta R_{k, i}$, and requiring that they are all real (i.e., $\operatorname{Im}\left\{\Delta R_{k, i}\right\}=0$ for $i=\{0,1, \ldots, N-1\}$ ). It is the property that the components of $\Delta R_{k}$ are all real that simplifies 
how they can be selected, and facilitates the design and analysis techniques employed in this paper. Full details of the use of DFT within ILC appear in, for example, [17].

\section{TRAJECTORY UPDATE SELECTION}

At the end of the $k$ th trial, $\Delta R_{k}$ must be chosen to satisfy (13). Having limited the set from which it may belong in the way described, it is easy to choose $\Delta r_{k}$ to minimize $\left\|\Delta r_{k}+e_{k}\right\|^{2}$ since there is no global constraint and the optimization may be conducted frequency-wise. Taking the inner product of the error, and applying Parseval's theorem

$$
\begin{aligned}
\left\|e_{k}^{*}\right\|^{2}= & \left\|\Delta r_{k}+e_{k}\right\|^{2} \\
= & \left\|\Delta r_{k}\right\|^{2}+\left\|e_{k}\right\|^{2}+2 \operatorname{Re}\left\langle\Delta r_{k}, e_{k}\right\rangle \\
= & \frac{1}{N} \sum_{i=0}^{N-1} \Delta R_{k, i}^{2}+\frac{1}{N} \sum_{i=0}^{N-1}\left|E_{k, i}\right|^{2} \\
& +\frac{2}{N} \sum_{i=0}^{N-1} \Delta R_{k, i} \operatorname{Re}\left\{E_{k, i}\right\} \\
= & \frac{1}{N} \sum_{i=0}^{N-1}\left|E_{k, i}\right|^{2} \\
& +\frac{1}{N} \sum_{i=0}^{N-1} \Delta R_{k, i}\left(\Delta R_{k, i}+2 \operatorname{Re}\left\{E_{k, i}\right\}\right) .
\end{aligned}
$$

Each frequency component of the reference change, $\Delta R_{k, i}$, can be chosen to minimize this in order to best satisfy (13). The differential with respect to $\Delta R_{k}$ is

$$
\frac{\partial}{\partial \Delta R_{k}}\left\|\Delta r_{k}+e_{k}\right\|^{2}=\frac{2}{N} \sum_{i=0}^{N-1} \Delta R_{k, i}+\frac{2}{N} \sum_{i=0}^{N-1} \operatorname{Re}\left\{E_{k, i}\right\}
$$

This is minimized using

$$
\Delta R_{k, i}=-\alpha \operatorname{Re}\left\{E_{k, i}\right\}
$$

with $\alpha=1$, however values of $\alpha \in\left(\begin{array}{ll}0 & 1\end{array}\right]$ will be considered, in order to better examine the transition between the proposed case and the use of a static reference. The optimal value of (13) on trial $k$ is accordingly given by

$$
\begin{aligned}
\left\|\Delta r_{k}+e_{k}\right\|^{2}= & \frac{1}{N} \sum_{i=0}^{N-1}\left|E_{k, i}\right|^{2} \\
& -\alpha(2-\alpha) \frac{1}{N} \sum_{i=0}^{N-1}\left(\operatorname{Re}\left\{E_{k, i}\right\}\right)^{2} \\
< & \left\|e_{k}\right\|^{2} \quad \text { iff } \exists i \text { s.t. } \operatorname{Re}\left\{E_{k, i}\right\} \neq 0
\end{aligned}
$$

since $0<\alpha(2-\alpha) \leq 1$. Note that, in the time domain, the reference is being updated using

$$
r_{k+1}=r_{k}+\Delta r_{k}
$$

which is selected to expediate the learning of the final trajectory. It is always possible to ensure $\left\|\Delta r_{k}+e_{k}\right\|^{2} \leq\left\|e_{k}\right\|^{2}$ since

$$
\begin{aligned}
& \left\|\Delta r_{k}+e_{k}\right\|^{2}-\left\|e_{k}\right\|^{2} \\
& =-\alpha(2-\alpha) \frac{1}{N} \sum_{i=0}^{N-1}\left(\operatorname{Re}\left\{E_{k, i}\right\}\right)^{2} \\
& <0 \quad \text { iff } \exists i \text { s.t. } \operatorname{Re}\left\{E_{k, i}\right\} \neq 0 .
\end{aligned}
$$

From (7) the error norm is given by

$$
\begin{aligned}
\left\|e_{k+1}\right\|^{2} & =\left\|\frac{\left(\Delta r_{k}+e_{k}\right)}{e_{k}}(I-G K) \star e_{k}\right\|^{2} \\
& \leq\left\|\frac{\left(\Delta r_{k}+e_{k}\right)}{e_{k}}(I-G K)\right\|^{2}\left\|e_{k}\right\|^{2}
\end{aligned}
$$

in which the division, and multiplication, $\star$, are executed component-wise. The norm error ratio is

$$
\begin{aligned}
\frac{\left\|e_{k+1}\right\|^{2}}{\left\|e_{k}\right\|^{2}} \leq & \frac{1}{N} \sum_{i=0}^{N-1} \\
& \frac{\left(\left|E_{k, i}\right|^{2}-\alpha(2-\alpha)\left(\operatorname{Re}\left\{E_{k, i}\right\}\right)^{2}\right)\left|1-G_{i} K_{i}\right|^{2}}{\left|E_{k, i}\right|^{2}} \\
= & \frac{1}{N} \sum_{i=0}^{N-1}\left(1-\alpha(2-\alpha) \cos ^{2}\left(\angle E_{k, i}\right)\right)\left|1-G_{i} K_{i}\right|^{2}
\end{aligned}
$$

and the proposed objective-driven ILC has introduced the multiplier $\sqrt{1-\alpha(2-\alpha) \cos ^{2}\left(\angle E_{k, i}\right)} \leq 1$ on the $i$ th frequency component. This multiplier relaxes the monotonic convergence criterion given by (10). From (21) a sufficient condition to produce trial-to-trial error reduction is now

$$
\left(1-\alpha(2-\alpha) \cos ^{2}\left(\angle E_{k, i}\right)\right)\left|1-G_{i} K_{i}\right|^{2}<1
$$

for each frequency, $i$, so that

$$
\left|1-G_{i} K_{i}\right|<\frac{1}{1-\alpha(2-\alpha) \cos ^{2}\left(\angle E_{k, i}\right)} .
$$

Increasing $\alpha$ reduces the denominator and so provides additional robustness with respect to the constantly modified reference.

\section{CONVERGENCE}

In this section, the monotonic convergence of the plant output to a fixed trajectory is established. Substituting (16) into the frequency-transformed ILC algorithm (4) (with $E_{k, i}^{*}=R_{k+1, i}-$ $\left.Y_{k, i}=R_{k+1, i}-G_{i} U_{k, i}\right)$ it is possible to find the real and imaginary components of the plant output. Using $\hat{\cdot}$ and ${ }^{-}$to denote 


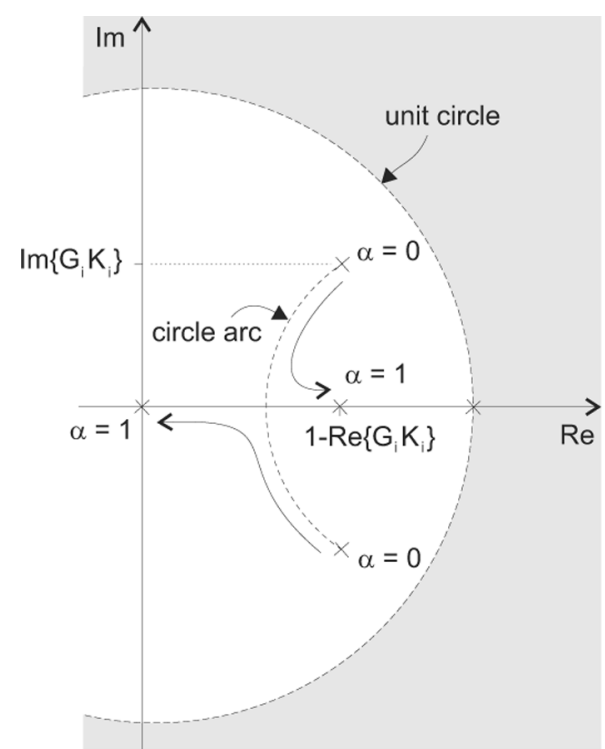

Fig. 1. Movement of eigenvalues (26) due to learning factor, $\alpha$.

$\operatorname{Re}\{\cdot\}$ and $\operatorname{Im}\{\cdot\}$, respectively, the resulting system can then be represented by

$$
\left[\begin{array}{c}
\bar{Y}_{k+1, i} \\
\hat{Y}_{k+1, i} \\
\hat{R}_{k+1, i}
\end{array}\right]=\Xi(i)\left[\begin{array}{c}
\bar{Y}_{k, i} \\
\hat{Y}_{k, i} \\
\hat{R}_{k, i}
\end{array}\right]+\left[\begin{array}{c}
\operatorname{Re}\left\{\mathrm{G}_{\mathrm{i}} \mathrm{K}_{\mathrm{i}}\right\} \overline{\mathrm{R}}_{0, \mathrm{i}} \\
-\operatorname{Im}\left\{\mathrm{G}_{\mathrm{i}} \mathrm{K}_{\mathrm{i}}\right\} \overline{\mathrm{R}}_{0, \mathrm{i}} \\
0
\end{array}\right]
$$

where $\Xi(i)$ is given by

$$
\left[\begin{array}{ccc}
1-\operatorname{Re}\left\{\mathrm{G}_{\mathrm{i}} \mathrm{K}_{\mathrm{i}}\right\} & -(1-\alpha) \operatorname{Im}\left\{\mathrm{G}_{\mathrm{i}} \mathrm{K}_{\mathrm{i}}\right\} & (1-\alpha) \operatorname{Im}\left\{\mathrm{G}_{\mathrm{i}} \mathrm{K}_{\mathrm{i}}\right\} \\
\operatorname{Im}\left\{\mathrm{G}_{\mathrm{i}} \mathrm{K}_{\mathrm{i}}\right\} & 1-(1-\alpha) \operatorname{Re}\left\{\mathrm{G}_{\mathrm{i}} \mathrm{K}_{\mathrm{i}}\right\} & (1-\alpha) \operatorname{Re}\left\{\mathrm{G}_{\mathrm{i}} \mathrm{K}_{\mathrm{i}}\right\} \\
0 & \alpha & 1-\alpha
\end{array}\right] .
$$

This system determines the behavior of the plant output and real component of the reference $\hat{R}$, however the imaginary component is not updated in order to satisfy the end-point conditions for every trial, so that $\bar{R}_{k, i}=\bar{R}_{0, i}$. The eigenvalues of $\Xi(i)$ are 1 and

$$
\begin{aligned}
\lambda_{1,2}(i)= & \left(1-\frac{\alpha}{2}\right)\left(1-\operatorname{Re}\left\{\mathrm{G}_{\mathrm{i}} \mathrm{K}_{\mathrm{i}}\right\}\right) \\
& \pm \sqrt{\left(\frac{\alpha}{2}\right)^{2}\left(1-\operatorname{Re}\left\{\mathrm{G}_{\mathrm{i}} \mathrm{K}_{\mathrm{i}}\right\}\right)^{2}-(1-\alpha)\left(\operatorname{Im}\left\{\mathrm{G}_{\mathrm{i}} \mathrm{K}_{\mathrm{i}}\right\}\right)^{2}}
\end{aligned}
$$

As $\alpha$ changes, their movement is shown in Fig. 1. When $\lambda_{1,2}(i)$ have complex components, they move along the circle arc shown. The derivation of this arc is given in the Appendix.

Now let $D(i)=\operatorname{diag}\left\{\lambda_{1}(i), \lambda_{2}(i), 1\right\}$, and $V(i)$ contain the corresponding eigenvectors such that $V(i) D(i) V^{-1}(i)$ represents the matrix diagonalization of $\Xi(i)$. It is straightforward to show that

$$
V^{-1}(i)\left[\begin{array}{c}
\operatorname{Re}\left\{\mathrm{G}_{\mathrm{i}} \mathrm{K}_{\mathrm{i}}\right\} \\
-\operatorname{Im}\left\{\mathrm{G}_{\mathrm{i}} \mathrm{K}_{\mathrm{i}}\right\} \\
0
\end{array}\right]=\left[\begin{array}{c}
v_{1}(i) \\
v_{2}(i) \\
0
\end{array}\right]
$$

where $v_{1}(i), v_{2}(i)$ may be complex (and if so occur as a conjugate pair). Then using the transformation

$$
\left[\begin{array}{c}
\tilde{\bar{Y}}_{k, i} \\
\tilde{\hat{Y}}_{k, i} \\
\tilde{\hat{R}}_{k, i}
\end{array}\right]=V^{-1}(i)\left[\begin{array}{c}
\bar{Y}_{k, i} \\
\hat{Y}_{k, i} \\
\hat{R}_{k, i}
\end{array}\right]
$$

allows (24) to be rewritten as the reduced order system

$$
\left[\begin{array}{c}
\tilde{\bar{Y}}_{k+1, i} \\
\hat{\hat{Y}}_{k+1, i}
\end{array}\right]=\left[\begin{array}{cc}
\lambda_{1}(i) & 0 \\
0 & \lambda_{2}(i)
\end{array}\right]\left[\begin{array}{c}
\tilde{\bar{Y}}_{k, i} \\
\hat{\hat{Y}}_{k, i}
\end{array}\right]+\left[\begin{array}{c}
v_{1}(i) \\
v_{2}(i)
\end{array}\right]
$$

together with $\tilde{\hat{R}}_{k, i}=\tilde{\hat{R}}_{0, i}$. As $k \rightarrow \infty,(29)$ converges asymptotically to

$$
\left[v_{1}(i) /\left(1-\lambda_{1}(i)\right), v_{2}(i) /\left(1-\lambda_{2}(i)\right)\right]^{T}
$$

and it therefore follows that (24) converges asymptotically to

$$
\left[\begin{array}{c}
\bar{Y}_{\infty, i} \\
\hat{Y}_{\infty, i} \\
\hat{R}_{\infty, i}
\end{array}\right]=V(i)\left[\begin{array}{c}
\frac{v_{1}(i)}{1-\lambda_{1}(i)} \\
\frac{v_{2}(i)}{1-\lambda_{2}(i)} \\
\hat{\hat{R}}_{0, i}
\end{array}\right]
$$

Here the converged values are given by

$$
\begin{aligned}
\bar{Y}_{\infty, i} & =\bar{R}_{0, i} \\
\hat{R}_{\infty, i} & =\frac{\xi}{\alpha+(1-\alpha) \operatorname{Re}\left\{\mathrm{G}_{\mathrm{i}} \mathrm{K}_{\mathrm{i}}\right\} \sec ^{2}\left(\angle \mathrm{G}_{\mathrm{i}} \mathrm{K}_{\mathrm{i}}\right)} \\
\hat{Y}_{\infty, i} & =\hat{R}_{\infty, i}
\end{aligned}
$$

where

$$
\begin{aligned}
\xi= & \alpha\left(\hat{Y}_{0, i}+\left(\bar{Y}_{0, i}-\bar{R}_{0, i}\right) \tan \left(\angle \mathrm{G}_{\mathrm{i}} \mathrm{K}_{\mathrm{i}}\right)\right) \\
& +(1-\alpha) \hat{R}_{0, i} \operatorname{Re}\left\{\mathrm{G}_{\mathrm{i}} \mathrm{K}_{\mathrm{i}}\right\} \sec ^{2}\left(\angle \mathrm{G}_{\mathrm{i}} \mathrm{K}_{\mathrm{i}}\right) .
\end{aligned}
$$

Note that the real and imaginary components of the output converge to $\hat{R}_{\infty, i}$ and $\bar{R}_{0, i}$, respectively, thereby satisfying the endpoint conditions (which are always satisfied by the reference). Let us now examine the nature of the convergence of the output to its final value. Since the plant output and reference both converge asymptotically, from (24), the convergence of the output to its final value is given by

$$
\left[\begin{array}{c}
\bar{Y}_{\infty, i}-\bar{Y}_{k+1, i} \\
\hat{Y}_{\infty, i}-\hat{Y}_{k+1, i} \\
\hat{R}_{\infty, i}-\hat{R}_{k+1, i}
\end{array}\right]=\Xi(i)\left[\begin{array}{c}
\bar{Y}_{\infty, i}-\bar{Y}_{k, i} \\
\hat{Y}_{\infty, i}-\hat{Y}_{k, i} \\
\hat{R}_{\infty, i}-\hat{R}_{k, i}
\end{array}\right]
$$

Applying the previous transformation gives

$$
V^{-1}(i)\left[\begin{array}{c}
\bar{Y}_{\infty, i}-\bar{Y}_{k+1, i} \\
\hat{Y}_{\infty, i}-\hat{Y}_{k+1, i} \\
\hat{R}_{\infty, i}-\hat{R}_{k+1, i}
\end{array}\right]=D(i) V^{-1}(i)\left[\begin{array}{c}
\bar{Y}_{\infty, i}-\bar{Y}_{k, i} \\
\hat{Y}_{\infty, i}-\hat{Y}_{k, i} \\
\hat{R}_{\infty, i}-\hat{R}_{k, i}
\end{array}\right] .
$$

Let the error now be redefined to be with respect to the final converged reference value (which clearly satisfies the end-point conditions), so that $\hat{E}_{k, i}^{*}=\hat{R}_{\infty, i}-\hat{Y}_{k, i}$ and $\bar{E}_{k, i}^{*}=\bar{R}_{\infty, i}-\bar{Y}_{k, i}$. This leads to

$$
\left[\begin{array}{c}
\tilde{\bar{E}}_{k+1, i}^{*} \\
\hat{\hat{E}}_{k+1, i}^{*} \\
0
\end{array}\right]=D(i)\left[\begin{array}{c}
\tilde{\bar{E}}_{k, i}^{*} \\
\hat{\hat{E}}_{k, i}^{*} \\
0
\end{array}\right]
$$


where we have used $\tilde{\hat{R}}_{k, i}=\tilde{\hat{R}}_{0, i}$. Therefore the eigenvalue at 1 and its associated eigenvector can be removed from the system, leading to

$$
\left[\begin{array}{c}
\bar{E}_{k+1, i}^{*} \\
\hat{E}_{k+1, i}^{*}
\end{array}\right]=\underbrace{V_{s}(i)\left[\begin{array}{cc}
\lambda_{1}(i) & 0 \\
0 & \lambda_{2}(i)
\end{array}\right] V_{s}^{-1}(i)}_{\Omega(i)}\left[\begin{array}{c}
\bar{E}_{k, i}^{*} \\
\hat{E}_{k, i}^{*}
\end{array}\right] .
$$

Here $V_{s}(i)$ is the upper right $2 \times 2$ minor of $V(i)$. It can be shown that $\bar{\sigma}(\Omega(i))=\left|1-G_{i} K_{i}\right|$. The condition for monotonic convergence to zero error is therefore

$$
\left|1-G_{i} K_{i}\right|<1
$$

which, for all frequencies up to Nyquist, is also a sufficient condition for (10) to be satisfied, and one which has been shown to be a necessary condition in practice for the static reference case [18]. The other singular value of (39) is also equal to $\left|1-G_{i} K_{i}\right|$ at $\alpha=0$ but reduces to zero as $\alpha$ increases to 1 , thereby significantly increasing the convergence rate. For $\alpha=1$, the interpretation of this is that learning immediately terminates for the mode that does not influence the point-to-point tracking objective, and continues only for the mode which does. Since, in ILC, learning acts in a wave moving from start to finish [18], this eliminates a significant portion of the transient response which slows convergence at the endpoint (since in ILC steady-state is never technically achieved). Moreover, by eliminating unnecessary learning, it is shown in the next section that substantial robustness advantages are gained.

The final output is given by $Y_{\infty}=Y_{0}+E_{0}^{*}$, where, for most initial reference and output values, the magnitude of $E_{0, i}^{*}$ reduces as $\alpha$ is increased. This can be seen from examination of its components for the values $\alpha=0$ and $\alpha=1$, given by

$$
\begin{aligned}
& \bar{E}_{0, i}^{*}=\bar{R}_{0, i}-\bar{Y}_{0, i} \text { for all } \alpha \\
& \bar{E}_{0, i}^{*}=\left\{\begin{array}{l}
\hat{R}_{0, i}-\hat{Y}_{0, i} \text { for } \alpha=0 \\
\left(\bar{Y}_{0, i}-\bar{R}_{0, i}\right) \tan \left(\angle G_{i} K_{i}\right) \text { for } \alpha=1 .
\end{array}\right.
\end{aligned}
$$

The condition for $\alpha=1$ to provide a reduction in $\left|E_{0, i}^{*}\right|$ compared with $\alpha=0$ is

$$
\tan \left(\angle G_{i} K_{i}\right)^{2}<\frac{\left(\hat{Y}_{0, i}-\hat{R}_{0, i}\right)^{2}}{\left(\bar{Y}_{0, i}-\bar{R}_{0, i}\right)^{2}}=\frac{1}{\tan \left(\angle\left(Y_{0, i}-R_{0, i}\right)\right)^{2}}
$$

which is equivalent to

$$
\begin{aligned}
& \min \left\{\left|\angle G_{i} K_{i}\right|,\left|\angle G_{i} K_{i} \pm \pi\right|,\left|\angle G_{i} K_{i} \pm 2 \pi\right|, \ldots\right\} \\
& \quad<\left|\frac{\pi(1+2 n)}{2}-\angle\left(Y_{0, i}-R_{0, i}\right)\right| \quad n \in \mathbb{Z} .
\end{aligned}
$$

To yield rapid convergence $G_{i} K_{i}$ must be placed close to +1 in the complex plane, so that (43) is satisfied for a large range of $Y_{0, i}-R_{0, i}$. When (43) is not satisfied, the faster convergence using $\alpha=1$ will still reduce the error norm below the $\alpha=0$ case within a small number of trials. If the final reference is for some reason unsatisfactory, its deviation from the original reference may be reduced through reduction of $\alpha$.

The frequency components of the first reference $R_{0, i}$ can be chosen to directly minimize $\left|E_{0, i}^{*}\right|$. From (41) and (42) this simply involves minimizing each component $\left|\bar{Y}_{0, i}-\bar{R}_{0, i}\right|$ which can be achieved by the following:

- experimenting with a range of initial plant inputs, $u_{0}$, and using the one which provides minimal $\left|\bar{Y}_{0, i}-\bar{R}_{0, i}\right|$ across all frequencies;

- after the first trial using an arbitrary reference, perform a global optimization over a suitable frequency range to minimize all $\left|\bar{Y}_{0, i}-\bar{R}_{0, i}\right|$ with respect to $R_{0}$ with the constraint that the reference still satisfies the point-to-point constraint. Then replace the old reference with its minimized counterpart, and proceed as normal.

\section{RoBUSTNESS}

It has been shown that $\alpha>0$ improves convergence by focusing on learning the error components which are associated with achieving the point-to-point objective. In this section it is shown that this also leads to greater robustness to model uncertainty. The system robustness can be examined by determining the range of plant uncertainty which may exist such that the error system (39) converges to zero. As in all such analysis, this is based on an assumption concerning the structure of the uncertainty. To illustrate this approach, a frequency-wise multiplicative plant uncertainty will be assumed, given by

$$
G_{i}=G_{0, i} M_{i} \quad i=0,1, \ldots, N-1
$$

where $G_{0, i}$ is the nominal plant. This is inserted in the expression for the eigenvalues (26), and the bound on the region of uncertainty which leads to their lying within the unit circle is found by setting

$$
\left(1-\frac{\alpha}{2}\right)\left(1-\operatorname{Re}\left\{\mathrm{G}_{0, \mathrm{i}} \mathrm{K}_{\mathrm{i}}\right\} \hat{M}_{i}+\operatorname{Im}\left\{\mathrm{G}_{0, \mathrm{i}} \mathrm{K}_{\mathrm{i}}\right\} \bar{M}_{i}\right) \pm \sqrt{\varsigma}=1
$$

with

$$
\begin{aligned}
\varsigma= & \left(\frac{\alpha}{2}\right)^{2}\left(1-\operatorname{Re}\left\{\mathrm{G}_{0, \mathrm{i}} \mathrm{K}_{\mathrm{i}}\right\} \hat{\mathrm{M}}_{\mathrm{i}}+\operatorname{Im}\left\{\mathrm{G}_{0, \mathrm{i}} \mathrm{K}_{\mathrm{i}}\right\} \overline{\mathrm{M}}_{\mathrm{i}}\right)^{2} \\
& -(1-\alpha)\left(\operatorname{Re}\left\{\mathrm{G}_{0, \mathrm{i}} \mathrm{K}_{\mathrm{i}}\right\} \overline{\mathrm{M}}_{\mathrm{i}}+\operatorname{Im}\left\{\mathrm{G}_{0, \mathrm{i}} \mathrm{K}_{\mathrm{i}}\right\} \hat{\mathrm{M}}_{\mathrm{i}}\right)^{2} .
\end{aligned}
$$

The solution to this prescribes a region of $\mathbb{C}$ in which the uncertainty must lie. In the current instance this is the union of sub-regions. The first is the solution to (45) if the LHS is assumed to have a non-zero complex component. The sign of the real component of (45) is unknown, but can be ignored if the magnitude of both sides of the expression are used. This results in

$$
\left(1-\frac{\alpha}{2}\right)^{2}\left(1-\operatorname{Re}\left\{\mathrm{G}_{0, \mathrm{i}} \mathrm{K}_{\mathrm{i}}\right\} \hat{\mathrm{M}}_{\mathrm{i}}+\operatorname{Im}\left\{\mathrm{G}_{0, \mathrm{i}} \mathrm{K}_{\mathrm{i}}\right\} \overline{\mathrm{M}}_{\mathrm{i}}\right)^{2}-\varsigma=1 .
$$

Following algebraic manipulation, this can be written as

$$
\begin{aligned}
\left(\hat{M}_{i}-\frac{\operatorname{Re}\left\{\mathrm{G}_{0, \mathrm{i}} \mathrm{K}_{\mathrm{i}}\right\}}{\left|\mathrm{G}_{0, \mathrm{i}} \mathrm{K}_{\mathrm{i}}\right|^{2}}\right)^{2}+\left(\bar{M}_{i}+\frac{\operatorname{Im}\left\{\mathrm{G}_{0, \mathrm{i}} \mathrm{K}_{\mathrm{i}}\right\}}{\left|\mathrm{G}_{0, \mathrm{i}} \mathrm{K}_{\mathrm{i}}\right|^{2}}\right)^{2} \\
=\frac{1}{(1-\alpha)\left|\mathrm{G}_{0, \mathrm{i}} \mathrm{K}_{\mathrm{i}}\right|^{2}}
\end{aligned}
$$

and represents a circle in the uncertainty space. The second subregion is that which results if the LHS of (45) is assumed to be real. Again, the sign of the first component is unknown, and 

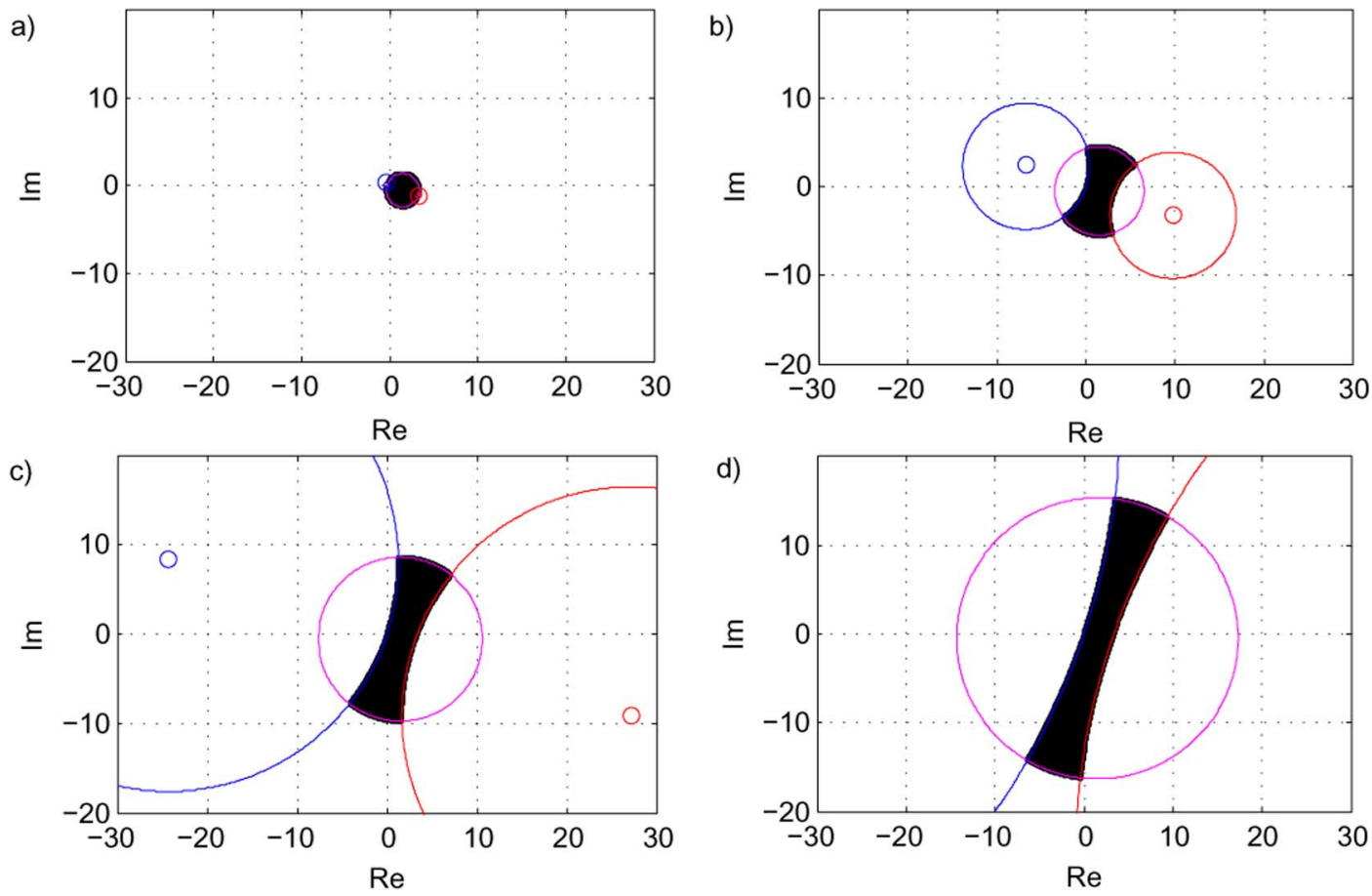

Fig. 2. Uncertainty region for which the eigenvalues $\lambda_{1,2}$ given by (26) lie in the unit circle, with $\operatorname{Re}\left\{\mathrm{G}_{\mathrm{i}} \mathrm{K}_{\mathrm{i}}\right\}=0.6, \operatorname{Im}\left\{\mathrm{G}_{\mathrm{i}} \mathrm{K}_{\mathrm{i}}\right\}=0.2$, for (a) $\alpha=0.3$, (b) $\alpha=0.9$, (c) $\alpha=0.97$, and (d) $\alpha=0.99$. The nominal system eigenvalues are: (a) $0.34 \pm 0.156 j$, (b) $0.051,0.389$, (c) $0.015,0.397$, and (d) $0.005,0.399$.

both cases must be considered. The magnitude of each term is therefore taken, which leads to

$\left|\left(1-\frac{\alpha}{2}\right)\left(1-\operatorname{Re}\left\{\mathrm{G}_{0, \mathrm{i}} \mathrm{K}_{\mathrm{i}}\right\} \hat{\mathrm{M}}_{\mathrm{i}}+\operatorname{Im}\left\{\mathrm{G}_{0, \mathrm{i}} \mathrm{K}_{\mathrm{i}}\right\} \overline{\mathrm{M}}_{\mathrm{i}}\right)\right|+| \pm \sqrt{\varsigma}|=1$.

After further manipulation, the first solution can be written as

$$
\begin{aligned}
\left(\hat{M}_{i}+\frac{\alpha \operatorname{Re}\left\{\mathrm{G}_{0, \mathrm{i}} \mathrm{K}_{\mathrm{i}}\right\}}{2\left|\mathrm{G}_{0, \mathrm{i}} \mathrm{K}_{\mathrm{i}}\right|^{2}(1-\alpha)}\right)^{2}+ & \left(\bar{M}_{i}-\frac{\alpha \operatorname{Im}\left\{\mathrm{G}_{0, \mathrm{i}} \mathrm{K}_{\mathrm{i}}\right\}}{2\left|\mathrm{G}_{0, \mathrm{i}} \mathrm{K}_{\mathrm{i}}\right|^{2}(1-\alpha)}\right)^{2} \\
& =\frac{\alpha^{2}}{4\left|\mathrm{G}_{0, \mathrm{i}} \mathrm{K}_{\mathrm{i}}\right|^{2}(1-\alpha)^{2}}
\end{aligned}
$$

and represents a circle. Similarly, the second solution can be written as

$$
\begin{aligned}
\left(\hat{M}_{i}+\frac{(3 \alpha-4) \operatorname{Re}\left\{\mathrm{G}_{0, \mathrm{i}} \mathrm{K}_{\mathrm{i}}\right\}}{2\left|\mathrm{G}_{0, \mathrm{i}} \mathrm{K}_{\mathrm{i}}\right|^{2}(1-\alpha)}\right)^{2}+ & \left(\bar{M}_{i}-\frac{(3 \alpha-4) \operatorname{Im}\left\{\mathrm{G}_{0, \mathrm{i}} \mathrm{K}_{\mathrm{i}}\right\}}{2\left|\mathrm{G}_{0, \mathrm{i}} \mathrm{K}_{\mathrm{i}}\right|^{2}(1-\alpha)}\right)^{2} \\
& =\frac{\alpha^{2}}{4\left|\mathrm{G}_{0, \mathrm{i}} \mathrm{K}_{\mathrm{i}}\right|^{2}(1-\alpha)^{2}}
\end{aligned}
$$

and again represents a circle. The intersection of the sub-regions with bounds given by (48), (50) and (51) therefore defines the region in which the plant uncertainty is bounded for any given $0 \leq \alpha \leq 1$. The transition between the cases $\alpha=0$ and $\alpha=1$ is illustrated by Fig. 2 in which the values $\operatorname{Re}\left\{\mathrm{G}_{0, \mathrm{i}} \mathrm{K}_{\mathrm{i}}\right\}=0.6$, $\operatorname{Im}\left\{\mathrm{G}_{0, \mathrm{i}} \mathrm{K}_{\mathrm{i}}\right\}=0.2$ have been assumed, together with an uncertainty region $-30 \leq \hat{M}_{i} \leq 30,-20 \leq \bar{M}_{i} \leq 20$. The circles are drawn and the shaded area gives the uncertainty that can be tolerated for convergence of the error system (39). The bounding circles represented by (48), (50), and (51) are illustrated in the figure by the magenta, blue, and red lines, respectively.

For $\alpha=0$ the first circle alone bounds the uncertainty region. As $\alpha$ increases so does its diameter, and the latter two circles move apart at a fixed angle, their diameters increasing so that their maximum separation distance is constant. Different values of $G_{i} K_{i}$ change the size and location of the first circle, and the angle at which the other two move apart. In all cases each value of $\alpha$ ensures stability for a different region of uncertainty space that was not provided by alternative $\alpha$ values, but it is clear that the largest region is associated with $\alpha=1$.

As discussed in Section IV, choosing $\alpha=1$ restricts learning to the frequency mode of (39) associated with achieving the point-to-point objective. The additional robustness demonstrated in this section is the result of not enforcing unnecessary learning in the direction of the other mode.

\section{MultiPle PoInT-to-Point Movements}

Only a single point-to-point task has so far been considered, comprising a movement from 0 to $r_{N}$ in $T$ seconds. Now let the reference be specified at a fixed number, $M$, of sample instants given by $I_{1}, I_{2}, \ldots, I_{M}$ with $I_{M}=N$. Let the prescribed values of the output at these instants be $J_{1}, J_{2}, \ldots, J_{M}$, with $J_{M}=$ $r_{N}$. It is proposed to choose the initial reference, $u^{*}$, to satisfy the optimization

$$
\text { minimize }\|u\|_{2}^{2} \quad \text { subject to } \quad \Phi G u=\Gamma
$$

where the $M \times N$ matrix $\Phi$ is defined by

$$
\begin{aligned}
\Phi_{i, p} & = \begin{cases}1, & i=1,2 \ldots M, \quad p=I_{i} \\
0, & \text { otherwise }\end{cases} \\
\Gamma & =\left[\begin{array}{llll}
J_{1} & J_{2} & \cdots & J_{M}
\end{array}\right]^{T} .
\end{aligned}
$$

An example of (52) using $T=10$ and $I_{1}=300, I_{2}=650$, and $I_{3}=T / T_{s}=1000$ with $J_{1}=7, J_{2}=-5$, and $J_{3}=10$ is now considered. Here $T_{s}$ denotes the sampling frequency. 
Since $I_{3}>I_{2}>I_{1}, \Phi$ is of the form (55), shown at the bottom of the page, so that the term $\Phi G$ appearing in (52) is given by $\Phi G=$ in (56) and (57), shown at the bottom of the page. Having performed the optimization, the corresponding initial reference $r_{1}=G u^{*}$ is shown in Fig. 7(a) using the plant described in Section VII. Returning to the general case, since the reference satisfies the desired point-to-point movement constraints, it can be updated by adding segments which are zero at samples $I_{1}, I_{2}, \ldots, I_{M}$. Therefore the single point-to-point update approach developed in Section III can be applied to each inter-point segment of the reference. The update for the $p$ th interval is accordingly given by

$$
r_{p, k+1}=r_{p, k}+\Delta r_{p, k} \quad p=1,2, \ldots, M
$$

where

$$
r_{p, k}=\left[\begin{array}{lll}
r_{k}\left(I_{p-1}+1\right) & \ldots & r_{k}\left(I_{p}\right)
\end{array}\right]^{T}
$$

with $I_{0}=1$. Likewise, the other signals are given by

$$
\begin{aligned}
y_{p, k} & =\left[\begin{array}{lll}
y_{k}\left(I_{p-1}+1\right) & \ldots & y_{k}\left(I_{p}\right)
\end{array}\right]^{T} \\
e_{p, k} & =\left[\begin{array}{lll}
e_{k}\left(I_{p-1}+1\right) & \ldots & e_{k}\left(I_{p}\right)
\end{array}\right]^{T} \\
\Delta r_{p, k} & =\left[\begin{array}{lll}
\Delta r_{k}\left(I_{p-1}+1\right) & \ldots & \Delta r_{k}\left(I_{p}\right)
\end{array}\right]^{T} .
\end{aligned}
$$

Since the $p$ th segment has $I_{p}-I_{p-1}$ elements, the reference update (16) is replaced by

$$
\Delta R_{p, k, i}=-\alpha \hat{E}_{p, k, i} \quad i=0,1, \ldots I_{p}-I_{p-1}-1 .
$$

Theorem: Application of the reference modification (63) and reference update (18), in combination with the error update (3) and ILC control law (4), for an $M$ segment point-to-point task, yields a system whose eigenvalues encompass those of the $M$ individual segments (which can then each be calculated using (26)).

Proof: The $i$ th frequency component of the $p$ th segment, $E_{p, k, i}$, is associated with the system (39), and its eigenvalues govern the convergence of the error in that segment. To find the contribution of each of the $M$ segments to the overall error, $e_{k}$, the $i$ th frequency component of $e_{k}$ is written as

$$
E_{k, i}=\sum_{n=0}^{N-1} e_{k} e^{-j 2 \pi n i / N}
$$

$$
\begin{aligned}
= & \sum_{p=1}^{M}\left(\sum_{n=I_{p-1}}^{I_{p}-1} e_{p, k} e^{-j 2 \pi n i / N}\right) \\
= & \sum_{p=1}^{M}\left(\sum_{n=0}^{I_{p}-I_{p-1}-1} e_{p, k} e^{-j 2 \pi\left(n+I_{p-1}\right) i / N}\right) \\
= & \sum_{p=1}^{M}\left(e^{-j 2 \pi I_{p-1} i / N} \sum_{n=0}^{I_{p}-I_{p-1}-1} e_{p, k} e^{-j 2 \pi n i / N}\right) \\
= & \sum_{p=1}^{M} e^{-j 2 \pi I_{p-1} i / N} E_{p, k, i\left(I_{p}-I_{p-1}\right) / N}
\end{aligned}
$$

where the DFT components of the $p$ th segment are given by

$$
E_{p, k, i}=\sum_{n=0}^{I_{p}-I_{p-1}-1} e_{p, k} e^{-j 2 \pi n i /\left(I_{p}-I_{p-1}\right)}
$$

so each frequency component of $e_{k}$ is a sum of frequencies from the $M$ segments. The $i$ th component of $e_{k+1}$ is

$$
E_{k+1, i}=\left[\begin{array}{c}
e^{-j 2 \pi I_{0} i / N} \\
e^{-j 2 \pi I_{1} i / N} \\
\vdots \\
e^{-j 2 \pi I_{M-1} i / N}
\end{array}\right]^{T}\left[\begin{array}{c}
E_{1, k+1, i\left(I_{1}-I_{0}\right) / N} \\
E_{2, k+1, i\left(I_{2}-I_{1}\right) / N} \\
\vdots \\
E_{M, k+1, i\left(I_{M}-I_{M-1}\right) / N}
\end{array}\right]
$$

Each segment error frequency component is governed by the system (39), which, using the current notation, is given by

$$
\left[\begin{array}{l}
\bar{E}_{p, k+1, l}^{*} \\
\hat{E}_{p, k+1, l}^{*}
\end{array}\right]=\Omega(l)\left[\begin{array}{l}
\bar{E}_{p, k, l}^{*} \\
\hat{E}_{p, k, l}^{*}
\end{array}\right]
$$

with

$$
E_{p, k+1, l}=[j, 1]\left[\begin{array}{c}
\bar{E}_{p, k+1, l}^{*} \\
\hat{E}_{p, k+1, l}^{*}
\end{array}\right]
$$

where $l=i\left(I_{p}-I_{p-1}\right) / N$. Therefore (66) becomes

$$
\begin{aligned}
E_{k+1, i} & =\left[\begin{array}{c}
e^{-j 2 \pi I_{0} i / N} \\
e^{-j 2 \pi I_{1} i / N} \\
\vdots \\
e^{-j 2 \pi I_{M-1} i / N}
\end{array}\right]^{T} \Upsilon X_{k+1} \\
X_{k+1} & =\Lambda(i) X_{k}
\end{aligned}
$$

$$
\Phi=\left[\begin{array}{cccccccccccc}
0 & \cdots & 0 & 1 & 0 & \cdots & \cdots & \cdots & \cdots & \cdots & \cdots & 0 \\
0 & \cdots & \cdots & \cdots & \cdots & \cdots & 0 & 1 & 0 & \cdots & \cdots & 0 \\
0 & \cdots & \cdots & \cdots & \cdots & \cdots & \cdots & \cdots & \cdots & \cdots & 0 & 1
\end{array}\right]
$$

$$
\begin{aligned}
& {\left[\begin{array}{cccccccccccc}
C A^{299} B & \ldots & C A B & C B & 0 & \ldots & \ldots & \ldots & \ldots & \ldots & \ldots & 0 \\
C A^{649} B & \ldots & \ldots & \ldots & \ldots & \ldots & C A B & C B & 0 & \ldots & \ldots & 0 \\
C A^{999} B & \ldots & \ldots & \ldots & \ldots & \ldots & \ldots & \ldots & \ldots & \ldots & C A B & C B
\end{array}\right]} \\
& \Gamma=\left[\begin{array}{lll}
7 & -5 & 10
\end{array}\right]^{T}
\end{aligned}
$$




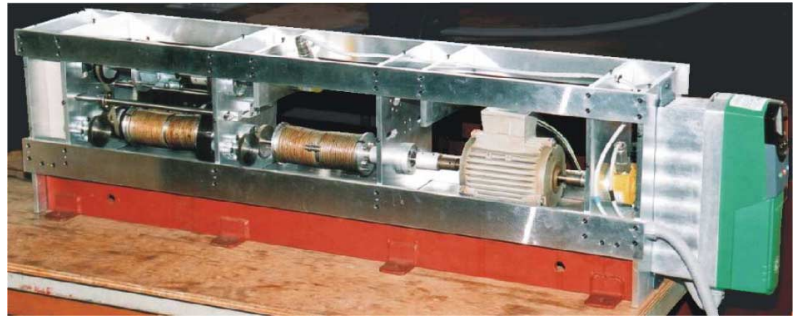

Fig. 3. Non-minimum phase experimental test facility.

with $X_{k+1} \in \mathbb{R}^{2 M}$ containing all the error components

$$
\begin{aligned}
\Upsilon= & {\left[\begin{array}{ccccccc}
j & 1 & 0 & 0 & \ldots & 0 & 0 \\
0 & 0 & j & 1 & \ldots & 0 & 0 \\
\vdots & \vdots & \vdots & \vdots & \ddots & \vdots & \vdots \\
0 & 0 & 0 & 0 & \ldots & j & 1
\end{array}\right] } \\
\Lambda(i)= & \operatorname{diag}\left\{\Omega\left(i\left(I_{1}-I_{0}\right) / N\right), \Omega\left(i\left(I_{2}-I_{1}\right) / N\right), \ldots,\right. \\
& \left.\Omega\left(i\left(I_{M}-I_{M-1}\right) / N\right)\right\} .
\end{aligned}
$$

As $\Lambda(i)$ is block diagonal, the eigenvalues of this system are

$$
\begin{array}{r}
\lambda(\Lambda(i))=\left\{\lambda\left(\Omega\left(i\left(I_{1}-I_{0}\right) / N\right)\right), \lambda\left(\Omega\left(i\left(I_{2}-I_{1}\right) / N\right)\right), \ldots,\right. \\
\left.\lambda\left(\Omega\left(i\left(I_{M}-I_{M-1}\right) / N\right)\right)\right\}
\end{array}
$$

where $\lambda(W)$ represents the set of all eigenvalues of $W$. Note that, from (39), $\lambda(\Omega(l))=\left\{\lambda_{1}(l), \lambda_{2}(l)\right\}$.

Hence the multiple point-to-point system inherits the eigenvalues and associated convergence and robustness properties of the single point-to-point systems which govern the behavior of each segment. For the case $\alpha=1$ the bound on the error, $e_{k}$, is

$$
\begin{aligned}
\left\|\Delta r_{k}+e_{k}\right\|^{2} & =\frac{1}{N} \sum_{p=1}^{M}\left\|\Delta r_{p, k}+e_{p, k}\right\|^{2} \\
& =\frac{1}{N} \sum_{p=1}^{M}\left(\left(I_{p}-I_{p-1}\right) \sum_{i=0}^{N-1}\left(\operatorname{Im}\left\{\mathrm{E}_{\mathrm{p}, \mathrm{k}, \mathrm{i}}\right\}\right)^{2}\right) \\
& <\frac{1}{N} \sum_{p=1}^{M}\left(\left(I_{p}-I_{p-1}\right) \sum_{i=0}^{N-1}\left|E_{p, k, i}\right|^{2}\right) \\
& <\left\|e_{k}\right\|^{2} \text { iff } \exists i, p \text { s.t. } \hat{E}_{p, k, i} \neq 0 .
\end{aligned}
$$

The final trajectory for the multiple point-to-point task is constructed from the final trajectories of each segment. For the $p$ th segment, the $q$ th frequency components are given by (32) and (34) with $i=q\left(I_{p}-I_{p-1}\right) / N$. This means the discussion in Section IV related to the bound on the final output is directly relevant.

Since it is often necessary for a system to perform a task at the final point-to-point position, it is desirable that the plant output come to rest at time $T$. This can be achieved by choosing the final segment of the initial reference to have the desired qualities (inserting an additional point $\left\{I_{M-1}, J_{M-1}\right\}$ if necessary), and reducing the value of $\alpha$ for this last section such that the change in reference is limited.

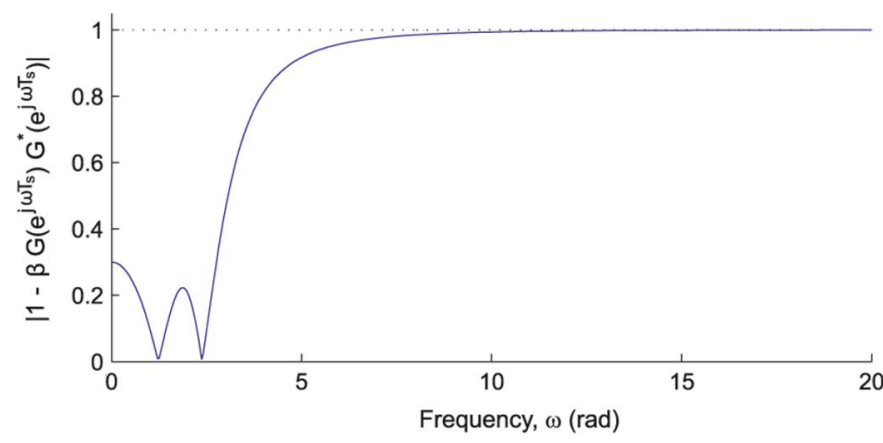

Fig. 4. $\left|1-\beta G\left(e^{j \omega T_{s}}\right) G^{*}\left(e^{j \omega T_{s}}\right)\right|$ for the non-minimum phase test facility using $\beta=0.7$.

\section{EXPERIMENTAL RESULTS}

The non-minimum phase test facility used to provide the experimental results is shown in Fig. 3, and has previously been used to evaluate a number of both ILC and repetitive control (RC) schemes (see [19], [20] for details). It consists of a rotary mechanical system of inertias, dampers, torsional springs, a timing belt, pulleys and gears. A 1000 pulse/rev encoder records the output shaft position and a standard squirrel cage induction motor supplied by an inverter, operating in variable voltage variable frequency (VVVF) mode, drives the load. The plant uses a PID loop in order to act as a prestabiliser, and the resulting closed-loop system constitutes the system to be controlled. The system can be represented using the continuous time plant transfer function

$$
G(s)=\frac{165.95(4-s)}{s^{4}+21.5 s^{3}+170.28 s^{2}+368.52 s+663.82}
$$

which has been identified in previous work [19]. The adjoint ILC algorithm is selected as a well known member of the class considered, and is given in discrete form by

$$
u_{k+1}(z)=u_{k}(z)+\beta G^{*}(z) e_{k}(z)
$$

where $G^{*}(z)$ is the adjoint of the plant model used (see [21] for theoretical background). An attractive feature of the method is that, with a sufficiently small positive scalar multiplier, $\beta$, it is guaranteed to satisfy the condition for monotonic convergence over all frequencies, and hence ensure a satisfactory transient response [22]. Since $K=\beta G^{*}$, the static reference monotonic convergence criterion corresponding to (10) in this case is

$$
\left\|I-\beta G G^{*}\right\|^{2}<1 .
$$

The corresponding frequency-wise condition (40) is

$$
\left|1-\beta G_{i} G_{i}^{*}\right|=\left.|1-\beta| G_{i}\right|^{2} \mid<1
$$

and is shown in Fig. 4, where the left-hand side approaches, but never reaches, 1 . This dictates the convergence of each frequency component, since the largest singular value of the error system (39) is given by $\left|1-G_{i} K_{i}\right|$. As discussed in Section IV, in the case of $\alpha=0$ this determines both singular values of the error system (39), however increasing $\alpha$ causes one of them to decrease to zero thereby increasing the convergence speed. 


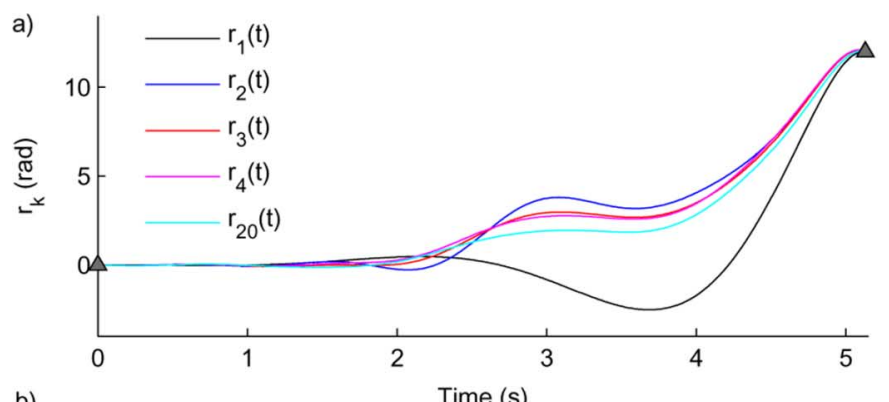

b)
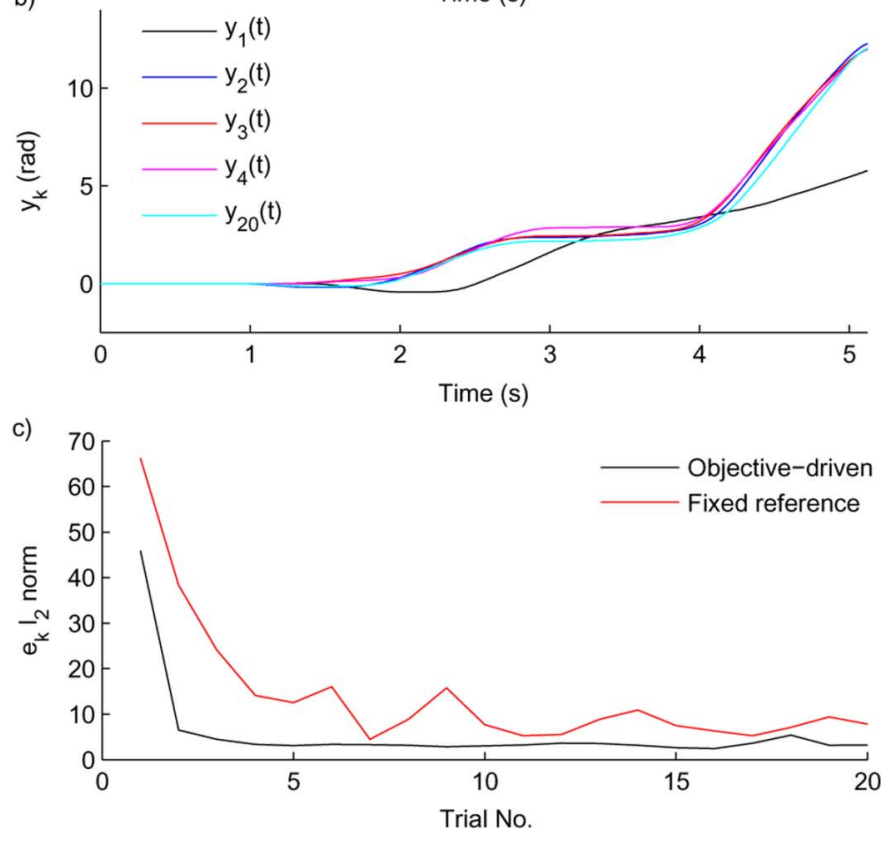

Fig. 5. Objective-driven ILC using the adjoint algorithm with $\beta=0.7, \alpha=1$, showing (a) reference evolution, (b) plant output, and (c) error norm $\left\|e_{k}\right\|$.

In the following experimental tests, a sampling period of $T_{s}=$ $0.01 \mathrm{~s}$ has been used. The adjoint ILC algorithm is one of the methods used in the stroke rehabilitation programme described in the introduction.

\section{A. Single Point-to-Point Task}

Fig. 5 shows experimental tracking results using the adjoint algorithm with $\beta=0.7, \alpha=1$, and the values $T=5.12 \mathrm{~s}$ and $r_{N}=12 \mathrm{rad}$. In the single point-to-point case, $M=1$, which leads to values of $I_{1}=512$ and $J_{1}=r_{N}$. The ILC algorithm is implemented using (4), and the reference is updated using (18) and (63) (which for the single point-to-point case equates to (16)), the latter operation being conducted in the frequency domain. Fig. 5(a) shows the reference quickly converges to a fixed signal which is markedly different from the reference used during the first trial. Fig. 5(b) shows the plant output over the course of the same trials, and Fig. 5(c) shows the error norm. It is clear that high accuracy tracking is achieved within five trials. For comparison, the error norm when using a static reference (equal to $r_{1}$ ) is also shown in the figure, and it can be seen that the objective-driven approach produces more accurate tracking in a reduced number of trials. The motivation for the proposed technique was to ensure the plant output equaled the end-point value $r_{N}$ at time $T$. To examine whether this has been achieved,

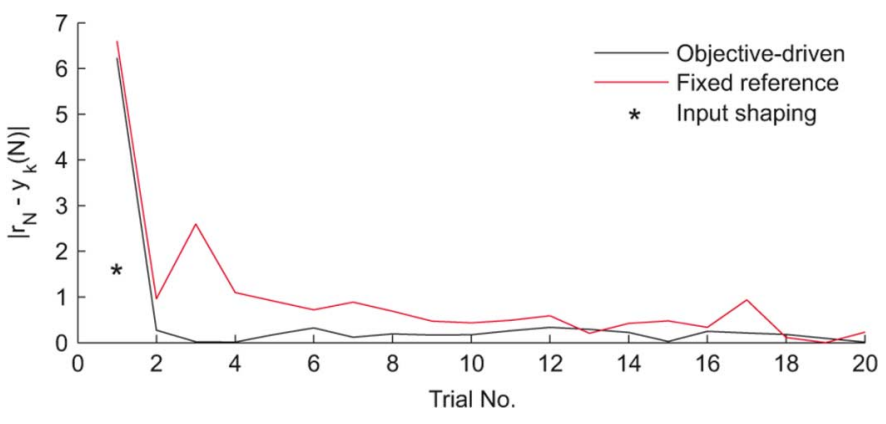

Fig. 6. Point-to-point error for objective-driven ILC using the adjoint algorithm with $\beta=0.7, \alpha=1$.
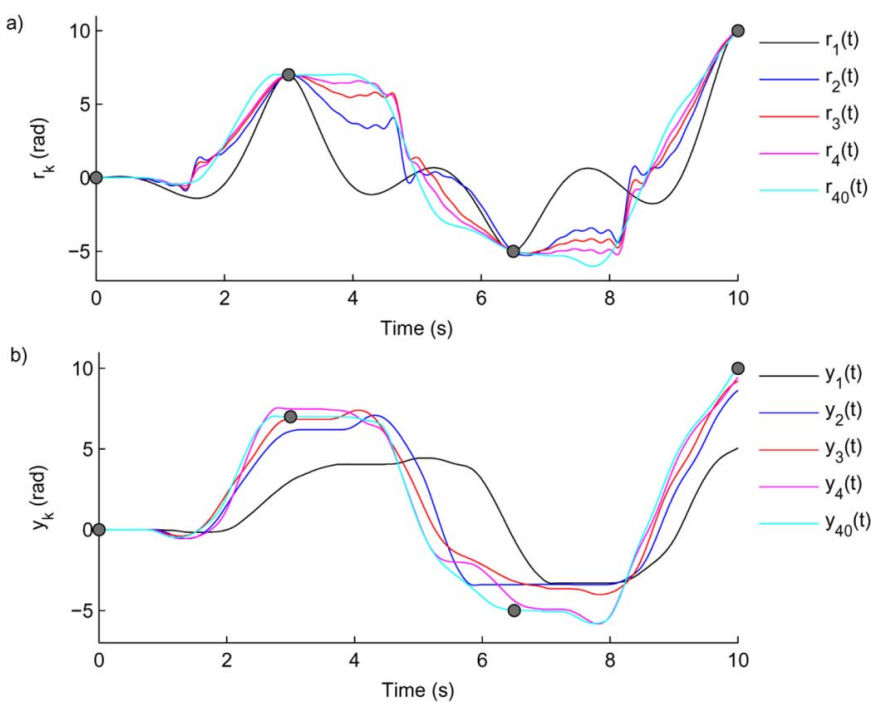

Fig. 7. Multiple objective-driven ILC using the adjoint algorithm with $\beta=0.6$ and $\alpha=1$, showing (a) reference evolution and (b) plant output.

Fig. 6 provides the final error value $\left|r_{N}-y_{k}(N)\right|$ using $\beta=0.7$. The results using a static reference are also shown, and it is clear that the proposed method has provided the capability to reach the end-point with greater accuracy in fewer trials.

\section{B. Multiple Point-to-Point Task}

The multiple point-to-point task developed in Section VI has also been tested on the non-minimum phase system, and the results are shown in Fig. 7. The reference update is shown in Fig. 7(a) and the plant output in (b) over 40 trials. The change in reference and plant output can be seen as $k$ increases, and the improvement in tracking of the points $\left(I_{p}, J_{p}\right)$ is evident. The error norm in tracking the reference $r_{k}$ is given in Fig. 8(a). The total error at the three points comprising the point-to-point movement, given by $\sum_{p=1}^{3}\left|J_{p}-y_{k}\left(I_{p}\right)\right|$, is shown in Fig. 8(b). In both cases, non-repeatable disturbances causes trial-to-trial fluctuation, but the use of higher $\alpha$ values provides reduced learning transients and additional robustness, resulting in increased tracking accuracy. In order to provide comparative results, the well known input shaping technique from [23] has been applied. First the experimentally-based tuning method described in [24] has been used to optimize the performance of the three term controller specifically for point-to-point application. Then the input satisfying (52) has been calculated for 


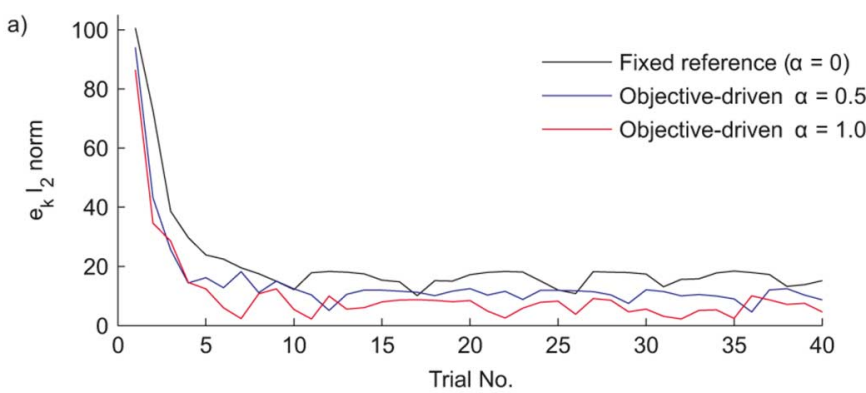

b)

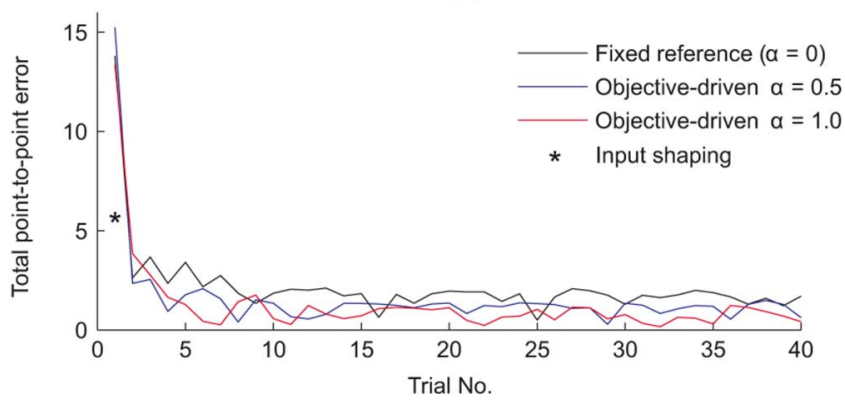

Fig. 8. Multiple objective-driven ILC using the adjoint algorithm with $\beta=0.6$ for a range of $\alpha$, showing (a) error norm $\left\|e_{k}\right\|$ and (b) total point-to-point error.

the new plant model. Input shaping has then been implemented using two impulses to remove residual vibration whilst still satisfying the point-to-point objective for the nominal model. Experimental results show that this leads to reasonable accuracy, as reflected by the total point-to-point errors that are included in Figs. 6 and 8 for the single and multiple cases, respectively. However, the results are significantly less accurate than the proposed iterative method.

\section{CONCLUSION}

A novel method of improving the performance of ILC when applied to point-to-point movements has been developed. The approach is based upon updating the reference between successive trials, and its convergence and robustness properties have been examined both theoretically and experimentally.

The technique's ability to vary the reference from trial to trial distinguishes it from traditional point-to-point motion strategies such as Input Shaping. This also separates it from previous applications of ILC to point-to-point motion control [12], [11], [10] which also assume a static reference. Moreover, whilst also providing the ability to learn from experience gained over previous trials of the task, the proposed scheme also has the benefit of being suitable for application to a broad class of existing ILC laws.

Having verified the approach using an electromechanical system, it can next be applied to ongoing research in the area of stroke rehabilitation, to assist patients in the performance of reaching tasks with their impaired arms using electrical stimulation. In this setting, the ability for the system dynamics to influence the path taken between points is of prime importance since it enhances the potential effectiveness of treatment. Experimental results will also be collected using a gantry robot system to verify the practical benefits of the approach in the field of robotics and automation.
Future work will attempt to remove the limitation that the change in reference must belong to the set of harmonic sinewaves. This will significantly complicate the analysis, but is expected to lead to further performance improvement. Focus will also be placed on extending the technique to allow variation in the time-points at which the movement attains the prescribed output values. Extension of the approach for application to nonlinear systems will then be formulated, with the addition of other constraints which govern the manner in which the task is performed.

\section{APPENDIX A \\ DERIVATION OF EIGENVALUE ARCS}

To derive the equation of the circle on which the eigenvalues of (24) move, the real and imaginary components of (26) are first written as $x$ and $y$, respectively, so that

$\begin{aligned} x & =\left(1-\frac{\alpha}{2}\right)\left(1-\operatorname{Re}\left\{\mathrm{G}_{\mathrm{i}} \mathrm{K}_{\mathrm{i}}\right\}\right) \\ y^{2} & =\left(\frac{\alpha}{2}\right)^{2}\left(1-\operatorname{Re}\left\{\mathrm{G}_{\mathrm{i}} \mathrm{K}_{\mathrm{i}}\right\}\right)^{2}-(1-\alpha)\left(\operatorname{Im}\left\{\mathrm{G}_{\mathrm{i}} \mathrm{K}_{\mathrm{i}}\right\}\right)^{2} .\end{aligned}$

Then substitute $\alpha$ from (78) into (79) to obtain

$$
\begin{aligned}
y^{2}= & \left(1-\frac{x}{\left(1-\operatorname{Re}\left\{\mathrm{G}_{\mathrm{i}} \mathrm{K}_{\mathrm{i}}\right\}\right)}\right)^{2}\left(1-\operatorname{Re}\left\{\mathrm{G}_{\mathrm{i}} \mathrm{K}_{\mathrm{i}}\right\}\right)^{2} \\
& -\left(\frac{2 x}{\left(1-\operatorname{Re}\left\{\mathrm{G}_{\mathrm{i}} \mathrm{K}_{\mathrm{i}}\right\}\right)}-1\right)\left(\operatorname{Im}\left\{\mathrm{G}_{\mathrm{i}} \mathrm{K}_{\mathrm{i}}\right\}\right)^{2} \\
= & x^{2}-2 x\left(\left(1-\operatorname{Re}\left\{\mathrm{G}_{\mathrm{i}} \mathrm{K}_{\mathrm{i}}\right\}\right)+\frac{\left(\operatorname{Im}\left\{\mathrm{G}_{\mathrm{i}} \mathrm{K}_{\mathrm{i}}\right\}\right)^{2}}{\left(1-\operatorname{Re}\left\{\mathrm{G}_{\mathrm{i}} \mathrm{K}_{\mathrm{i}}\right\}\right)}\right) \\
& +\left(\left(\operatorname{Im}\left\{\mathrm{G}_{\mathrm{i}} \mathrm{K}_{\mathrm{i}}\right\}\right)+\left(1-\operatorname{Re}\left\{\mathrm{G}_{\mathrm{i}} \mathrm{K}_{\mathrm{i}}\right\}\right)^{2}\right) .
\end{aligned}
$$

This describes a circle with centre

$$
\begin{aligned}
c & =\left(1-\operatorname{Re}\left\{\mathrm{G}_{\mathrm{i}} \mathrm{K}_{\mathrm{i}}\right\}\right)\left(1+\frac{\left(\operatorname{Im}\left\{\mathrm{G}_{\mathrm{i}} \mathrm{K}_{\mathrm{i}}\right\}\right)^{2}}{\left(1-\operatorname{Re}\left\{\mathrm{G}_{\mathrm{i}} \mathrm{K}_{\mathrm{i}}\right\}\right)^{2}}\right) \\
& >\left(1-\operatorname{Re}\left\{\mathrm{G}_{\mathrm{i}} \mathrm{K}_{\mathrm{i}}\right\}\right) \text { if } \operatorname{Im}\left\{\mathrm{G}_{\mathrm{i}} \mathrm{K}_{\mathrm{i}}\right\} \neq 0
\end{aligned}
$$

and radius

$$
\begin{aligned}
r & =\left|\operatorname{Im}\left\{\mathrm{G}_{\mathrm{i}} \mathrm{K}_{\mathrm{i}}\right\}\right| \sqrt{1+\frac{\left(\operatorname{Im}\left\{\mathrm{G}_{\mathrm{i}} \mathrm{K}_{\mathrm{i}}\right\}\right)^{2}}{\left(1-\operatorname{Re}\left\{\mathrm{G}_{\mathrm{i}} \mathrm{K}_{\mathrm{i}}\right\}\right)^{2}}} \\
& >\left|\operatorname{Im}\left\{\mathrm{G}_{\mathrm{i}} \mathrm{K}_{\mathrm{i}}\right\}\right| \text { if } \operatorname{Im}\left\{\mathrm{G}_{\mathrm{i}} \mathrm{K}_{\mathrm{i}}\right\} \neq 0 .
\end{aligned}
$$

The intersection with the real axis occurs at

$$
\begin{aligned}
c-r & =\left(1-\operatorname{Re}\left\{\mathrm{G}_{\mathrm{i}} \mathrm{K}_{\mathrm{i}}\right\}\right)\left(1+\frac{\left(\operatorname{Im}\left\{\mathrm{G}_{\mathrm{i}} \mathrm{K}_{\mathrm{i}}\right\}\right)^{2}}{\left(1-\operatorname{Re}\left\{\mathrm{G}_{\mathrm{i}} \mathrm{K}_{\mathrm{i}}\right\}\right)^{2}}\right) \\
& =-\left|\operatorname{Im}\left\{\mathrm{G}_{\mathrm{i}} \mathrm{K}_{\mathrm{i}}\right\}\right| \sqrt{1+\frac{\left(\operatorname{Im}\left\{\mathrm{G}_{\mathrm{i}} \mathrm{K}_{\mathrm{i}}\right\}\right)^{2}}{\left(1-\operatorname{Re}\left\{\mathrm{G}_{\mathrm{i}} \mathrm{K}_{\mathrm{i}}\right\}\right)^{2}}} \\
& =\frac{\left|1-\mathrm{G}_{i} K_{i}\right|}{1-\operatorname{Re}\left\{\mathrm{G}_{\mathrm{i}} \mathrm{K}_{\mathrm{i}}\right\}}\left(\left|1-\mathrm{G}_{\mathrm{i}} \mathrm{K}_{\mathrm{i}}\right|-\left|\operatorname{Im}\left\{\mathrm{G}_{\mathrm{i}} \mathrm{K}_{\mathrm{i}}\right\}\right|\right) .
\end{aligned}
$$


Since it is assumed $0 \leq\left(1-\operatorname{Re}\left\{\mathrm{G}_{\mathrm{i}} \mathrm{K}_{\mathrm{i}}\right\}\right)<1$

$$
\begin{aligned}
\left|1-G_{i} K_{i}\right| & \geq\left|\operatorname{Im}\left\{\mathrm{G}_{\mathrm{i}} \mathrm{K}_{\mathrm{i}}\right\}\right| \\
\left|1-G_{i} K_{i}\right|\left|\operatorname{Im}\left\{\mathrm{G}_{\mathrm{i}} \mathrm{K}_{\mathrm{i}}\right\}\right| & \geq\left(\operatorname{Im}\left\{\mathrm{G}_{\mathrm{i}} \mathrm{K}_{\mathrm{i}}\right\}\right)^{2} \\
\left(1-\operatorname{Re}\left\{\mathrm{G}_{\mathrm{i}} \mathrm{K}_{\mathrm{i}}\right\}\right)^{2} & \geq\left|1-G_{i} K_{i}\right|^{2} \\
& -\left|1-G_{i} K_{i}\right|\left|\operatorname{Im}\left\{\mathrm{G}_{\mathrm{i}} \mathrm{K}_{\mathrm{i}}\right\}\right|
\end{aligned}
$$

which leads to

$$
\frac{\left|1-G_{i} K_{i}\right|}{1-\operatorname{Re}\left\{\mathrm{G}_{\mathrm{i}} \mathrm{K}_{\mathrm{i}}\right\}}\left(\left|1-G_{i} K_{i}\right|-\left|\operatorname{Im}\left\{\mathrm{G}_{\mathrm{i}} \mathrm{K}_{\mathrm{i}}\right\}\right|\right) \leq 1-\operatorname{Re}\left\{\mathrm{G}_{\mathrm{i}} \mathrm{K}_{\mathrm{i}}\right\} \text {. }
$$

From (83) the intersection $c-r$ therefore occurs between 0 and $\left(1-\operatorname{Re}\left\{\mathrm{G}_{\mathrm{i}} \mathrm{K}_{\mathrm{i}}\right\}\right)$.

\section{REFERENCES}

[1] H. S. Ahn, Y. Chen, and K. L. Moore, "Iterative learning control: Brief survey and categorization," IEEE Trans. Syst., Man, Cybern. Pt. C, Appl. Rev., vol. 37, no. 6, pp. 1099-1121, Jun. 2007.

[2] K. L. Moore and A. Mathews, "Iterative learning control for systems with non-uniform trial length with applications to gas metal arc welding," presented at the 2nd Asian Control Conf., Seoul, Korea, 1997.

[3] S. Kawamura and N. Sakagami, "Analysis on dynamics of underwater robot manipulators basing on iterative learning control and time-scale transformation," in Proc. IEEE Int. Conf. Robot. Autom., Washington, DC, 2002, pp. 1088-1094.

[4] M. Grundelius and B. Bernhardsson, "Control of liquid slosh in an industrial packaging machine," presented at the IEEE Int. Conf. Control Appl., Kohala Coast, HI, 1999.

[5] A. G. Dharne and S. Jayasuriya, "Robust adaptive control of residual vibration in point-to-point motion of flexible bodies," J. Vibr. Control, vol. 13, no. 7, pp. 951-968, 2007.

[6] T. Singh and W. Singhose, "Tutorial on input shaping/time delay control of maneuvering flexible structures," in Proc. Amer. Control Conf., Anchorage, AK, 2002, pp. 1717-1731.

[7] B. G. Dijkstra, N. J. Rambaratsingh, C. Scherer, O. H. Bosgra, M. Steinbuch, and S. Kerssemakers, "Input design for optimal discrete time point-to-point motion of an industrial XY-positioning table," in Proc. 39th IEEE Conf. Dec. Control, Sydney, Australia, 2000, vol. 1, pp. 901-906.

[8] J. T. Belts, "Survey of numerical methods for trajectory optimization," J. Guid., Control, Dyn., vol. 21, no. 2, pp. 193-207, 1998.

[9] A. B. Doyle, "Algorithms and computational techniques for robot path planning," Ph.D. dissertation, Sch. Electron. Eng. Comput. Syst., Univ. Wales, Wales, U.K., 1995.

[10] J. van de Wijdeven and O. Bosgra, "Residual vibration suppression using hankel iterative learning control," Int. J. Rob. Nonlinear Control, vol. 18, pp. 1034-1051, 2008.

[11] J. Park, P. H. Chang, H. S. Park, and E. Lee, "Design of learning input shaping technique for residual vibration suppression in an industrial robot," IEEE/ASME Trans. Mechatron., vol. 11, no. 1, pp. 55-65, Feb. 2006.

[12] H. Ding and J. Wu, "Point-to-point control for a high-acceleration positioning table via cascaded learning schemes," IEEE Trans. Ind. Electron., vol. 54, no. 5, pp. 2735-2744, May 2007.

[13] C. T. Freeman, A. M. Hughes, J. H. Burridge, P. H. Chappell, P. L. Lewin, and E. Rogers, "Iterative learning control of FES applied to the upper extremity for rehabilitation," Control Eng. Pract., vol. 17, no. 3, pp. 368-381, 2009.

[14] A. M. Hughes, C. T. Freeman, J. H. Burridge, P. H. Chappell, P. L. Lewin, and E. Rogers, "Feasibility of iterative learning control mediated by functional electrical stimulation for reaching after stroke," $\mathrm{Neu}$ rorehabilitation Neural Repair, vol. 23, no. 6, pp. 559-568, 2009.

[15] D. A. Bristow, M. Tharayil, and A. G. Alleyne, "A survey of iterative learning control a learning-based method for high-performance tracking control," IEEE Control Syst. Mag., vol. 26, no. 3, pp. 96-114, Jun. 2006
[16] J. J. Hätönen, D. H. Owens, and K. L. Moore, “An algebraic approach to iterative learning control," Int. J. Control, vol. 77, no. 1, pp. 45-54, 2004.

[17] C. T. Freeman, P. L. Lewin, E. Rogers, J. J. Hätönen, and D. H. Owens, "Discrete Fourier transform based iterative learning control design for linear plants with experimental verification," ASME J. Dyn. Syst., Meas., Control, vol. 131, no. 3, pp. 031 006-1-031 006-10, 2009.

[18] R. W. Longman, "Iterative learning control and repetitive control for engineering practice," Int. J. Control, vol. 73, pp. 930-954, 2000.

[19] C. T. Freeman, P. L. Lewin, and E. Rogers, "Further results on the experimental evaluation of iterative learning control algorithms for nonminimum phase plants," Int. J. Control, vol. 80, no. 4, pp. 569-582, 2007.

[20] C. T. Freeman, P. L. Lewin, and E. Rogers, "Experimental evaluation of iterative learning control algorithms for non-minimum phase plants," Int. J. Control, vol. 78, no. 11, pp. 826-846, 2005.

[21] K. Furuta and M. Yamakita, "The design of learning control systems for multivariable systems," in Proc. IEEE Int. Symp. Intell. Control, Philadelphia, PA, 1987, pp. 371-376.

[22] K. Chen and R. W. Longman, "Stability issues using FIR filtering in repetitive control," Adv. Astronautical Sci., vol. 206, pp. 1321-1339, 2002.

[23] N. C. Singer and W. P. Seering, "Preshaping command inputs to reduce systems vibration," ASME J. Dyn. Syst., Meas., Control, vol. 112, no. 1, pp. 76-82, 1990.

[24] C. T. Freeman, "Experimental evaluation of iterative learning control on non-minimum phase plant," Ph.D. dissertation, Sch. Electron. Comput. Sci., Univ. Southampton, Southamptom, U.K., 2004.

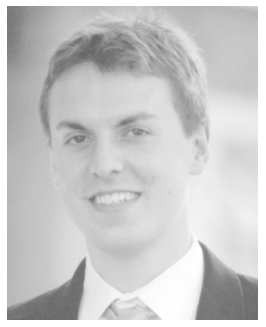

Chris Freeman received the B.Eng. degree in electromechanical engineering and the Ph.D. degree in applied control from the University of Southampton, Southampton, U.K., in 2000 and 2004, respectively.

From 2004 to 2007, he was a Post-Doctoral Researcher with the same university applying robotics and advanced control strategies to the field of upper limb stroke rehabilitation. In 2007, he was appointed Lecturer with the University of Southampton. His research is focussed on the development, application, and assessment of iterative learning and repetitive controllers within both the biomedical engineering domain and for application to industrial systems.

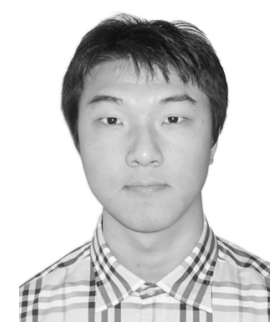

Zhonglun Cai received the B.Eng. degree in mechanical engineering from Jinan University in China, Jinan, China, in 2005 and the Ph.D. degree in electronic and electrical engineering from the University of Southampton, Southampton, U.K., in 2009.

From 2009, he started Post-Doctoral Research with the same university on iterative learning control applied to 3-D stroke rehabilitation. His research interests include iterative learning control applied to robotics and industrial applications.

Eric Rogers received the M.S. and Ph.D. degrees in control systems from The University of Sheffield, Sheffield, U.K., and the D.Sc. degree from The Queen's University of Belfast, Belfast, U.K., for research in control systems.

$\mathrm{He}$ is currently a Professor of Control Systems Theory and Design, School of Electronics and Computer Science.

Paul L. Lewin received the B.Sc. and Ph.D. degrees in electrical engineering from the University of Southampton, Southampton, U.K., in 1986 and 1994, respectively.

He joined the academic staff of the University of Southampton in 1989 and is currently Reader in Electrical Power Engineering within the School of Electronics and Computer Science. 